\title{
RESEARCH HIGHLIGHT New roles for RAD52 in DNA repair
}

\author{
Chaoyou Xue $^{1}$ and Eric C. Greene ${ }^{1}$ \\ Cell Research (2018) 28:1127-1128; https://doi.org/10.1038/s41422-018-0105-8
}

\begin{abstract}
Transcriptionally active genetic loci can be subject to the formation of DNA double strand breaks (DSBs) that arise due to the formation of R-loops. In a recent study published in Cell, Yasuhara and colleagues have revealed a remarkable new mechanism that allows cells to channel DSBs within active genes through a new repair pathway called transcriptionassociated homologous recombination repair.
\end{abstract}

Double strand breaks (DSBs) are one of the most dangerous forms of DNA damage because they can cause the gross chromosomal rearrangements that are hallmarks of human cancers. Transcription is a leading source of DSBs. ${ }^{1}$ During transcription, the nascent RNA can hybridize with the DNA to form R-loops (Fig. 1). Recent studies have revealed intriguing relationships betwee7n R-loops and homologous recombination $(\mathrm{HR}) .^{2-4}$ For instance, Ohle et al. demonstrated that efficient HR in $S$. pombe actually requires the formation of R-loops. ${ }^{2}$ Moreover, Rad52 was found to promote DSB repair in $S$. cerevisiae by allowing the cells to use the RNA as a template to guide DNA repair. ${ }^{3}$ Finally, Pomerantz and colleagues have defined two modes of RNA-directed DNA repair, both of which require RAD52. ${ }^{4}$ One mechanism involves RNA bridging to coordinate synapsis and ligation of DNA breaks, and the second mechanism uses RNA as a template for reverse transcription-dependent replacement of damaged DNA. ${ }^{4}$

In the newest study linking R-loops to DNA repair, Yasuhara and colleagues have found that human cells can channel DSBs formed within active genes through transcription-associated homologous recombination repair (TA-HRR; Fig. 1). ${ }^{5}$ They began by comparing the effects of ionizing radiation (IR) on cells that were undergoing normal transcription, or cells that had been treated with a transcription inhibitor. They then monitored DNA repair-related events by examining the formation and protein composition of DNA repair foci, and by looking for evidence of recombination based on the frequency of sister chromatid exchange (SEC). The formation of RPA and Rad51 foci indicates the existence of DSBs that are being repaired via HR. Cells that were not treated with the transcription inhibitor exhibited lots of RPA and Rad51 foci after exposure to IR. However, Yasuhara et al. observed a small, but statistically significant reduction in RPA and Rad51 foci when transcription was inhibited. ${ }^{5}$ Moreover, there was also a reduction in SCE for cells that were not transcriptionally active. These findings suggested that transcription was required for the repair of some DSBs.

Given the link between Rad52, R-loops and DSB repair in yeast, ${ }^{1-4}$ the authors sought to determine whether human RAD52 might participate in the repair of transcription-associated DSBs. To address this issue, the authors used CRISPR-Cas9 genome editing to knock out the RAD52 gene and then asked whether this knockout affected TA-HRR. The authors found reductions in RPA foci, RAD51 foci, and SCE in $\triangle R A D 52$ cells, which were comparable to the reductions observed in wild-type cells after inhibition of RNA synthesis. Remarkably, RAD51 recruitment to DSBs in transcriptionally active genes was RAD52 dependent, whereas RAD51 recruitment to transcriptionally inactive genes was not. This result pinpointed RAD52 as a critical factor for promoting HRmediated DSB repair in actively transcribed genes (Fig. 1). To further clarify the role of RAD52 in TA-HRR, they tested the effects of several key recombination factors that contribute to the decision as to whether a DSB will be repaired by non-homologous end joining (NHEJ) or HR. ${ }^{5}$ The key step in this decision is DNA end resection - DSBs that are not resected are repaired via NHEJ, whereas those that are resected get repaired via HR. 53BP1 and RIF1 inhibit resection, thus favoring NHEJ, while BRCA1 and CtPI counteract 53BP1 and RIF1 to promote HR. ${ }^{5}$ The authors showed that RAD52 and CtIP recruited BRCA1 to the DSBs, which antagonized the ability of RIF1 and 53BP1 to downregulate end resection (Fig. 1)

Given the high binding affinity of RAD52 on R-loops in vitro, it seemed reasonable to assume that RAD52 was in fact directly recruited to the $\mathrm{R}$-loops. To test this model, the authors used laser microbeam irradiation to generate DNA damage and then showed that GFP-tagged RAD52 rapidly accumulated at these sites. Remarkably, RAD52 accumulation was significantly reduced in cells overexpressing RNase $\mathrm{H} 1$, which is a nuclease that specifically cleaves the RNA strand within RNA-DNA hybrids, indicating that RAD52 accumulation was due to the presence of R-loops. As the final piece of the puzzle, Yasuhara et al. sought to determine how the R-loops were taken apart during repair. The resulting data demonstrated that $\mathrm{R}$-loop clearance was delayed in cells lacking RAD52. The authors next turned their attention to two nucleases, XPG and XPF. Both nucleases participate in nucleotide excision repair where they excise damaged DNA bases, ${ }^{6}$ but recent work from Sollier et al. had also implicated XPG and XPF as participants in R-loop processing. ${ }^{7}$ Remarkably, XPG was necessary for R-loop clearance, whereas XPF was not required, indicating that RAD52 and XPG work together to remove the R-loops (Fig. 1).

Rad52 is among the most important proteins for genome maintenance in yeast, but it seemed mysteriously inconsequential for DNA repair in higher eukaryotes. However, this viewpoint is quickly changing. The first hints suggesting an important role for RAD52 came from the seminal study of Powell and colleagues, who showed that RAD52 was synthetically lethal with the breast cancer gene BRCA2. ${ }^{8}$ Next, came studies showing that RAD52 was necessary for efficient DNA synthesis in cells subject to replication stress. ${ }^{9,10}$ The work of Yasuhara et al. is the latest of these exciting studies to highlight the importance of human RAD52 for maintaining genome integrity. 


\section{Transcriptionally Inactive}
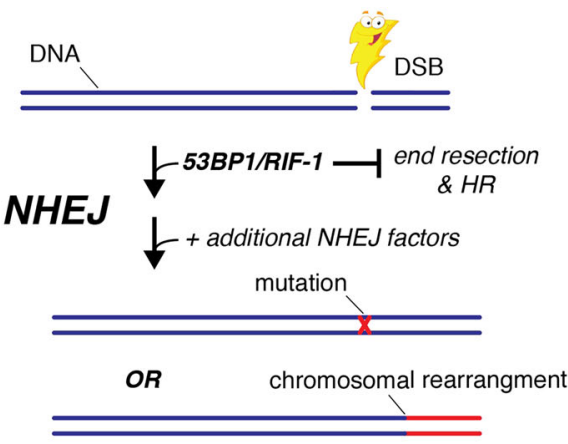

error-prone repair

\section{Transcriptionally Active}
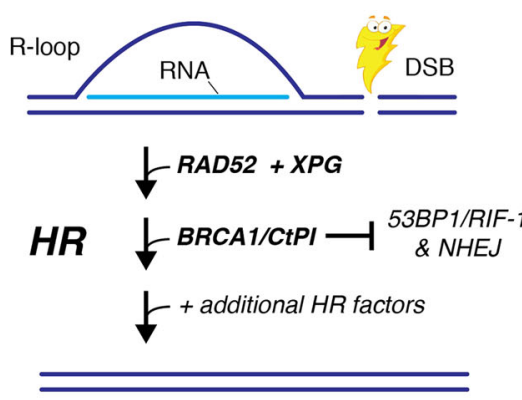

error-free repair

Fig. 1 Transcription-associated homologous recombination repair. DSBs that form in transcriptionally inactive regions of the genome are repaired by NHEJ, which has the potential to introduce mutations or chromosome rearrangement (left panel). In contrast, transcriptionally active regions are repaired by error-free HR, which involves recognition of R-loops by RAD52 and also requires the participation of BRCA1, CtIP and XPG (right panel)

\section{ADDITIONAL INFORMATION}

Conflict of interests: The authors declare that they have no conflict of interest.

\section{REFERENCES}

1. Skourti-Stathaki, K. \& Proudfoot, N. J. Genes Dev. 28, 1384-1396 (2014).

2. Ohle, C. et al. Cell 167, 1001-1013 (2016)

3. Keskin, H. et al. Nature 515, 436-439 (2014).
4. McDevitt, S. et al. Nat. Commun. 9, 1091 (2018)

5. Yasuhara, T. et al. Cell 175, 558-570 (2018).

6. Nouspikel, T. Cell. Mol. Life Sci. 66, 994-1009 (2009).

7. Sollier, J. et al. Mol. Cell 56, 777-785 (2014).

8. Feng, Z. et al. Proc. Natl Acad. Sci. USA 108, 686-691 (2011).

9. Bhowmick, R., Minocherhomji, S. \& Hickson, I. D. Mol. Cell 64, 1117-1126 (2016).

10. Sotiriou, S. K. et al. Mol. Cell 64, 1127-1134 (2016). 